\title{
ORTHOPAIRS: A SIMPLE AND WIDELY USED WAY TO MODEL UNCERTAINTY
}

\author{
D. CIUCCI
}

\begin{abstract}
The term orthopair is introduced to group under a unique definition different ways used to denote the same concept. Some orthopair models dealing with uncertainty are analyzed both from a mathematical and semantical point of view, outlining similarities and differences among them. Finally, lattice operations on orthopairs are studied and a survey on algebraic structures is provided.
\end{abstract}

\section{INTRODUCTION}

Uncertainty in information can be caused by different factors: incompleteness, imprecision, vagueness, differing standpoints, granularity. Several approaches have been defined to represent and manage all these defects: starting from probability theories in the XVIIth century, the field assumed a great importance in computer science and artificial intelligence [29]. Among these approaches we have probability theory, possibility theory, Dempster-Shafer theory, fuzzy sets, rough sets, formal concept analysis, ... and derived and mixing paradigms, such as fuzzy rough sets, imprecise probabilities.

Here, we desire to put in evidence the links among several approaches to uncertainty and describe a unique framework for these different models which makes easy their comparison and enables a common study on operations and algebraic structures. We decided to use the term orthopair (and equivalently nested pair) in order to concentrate purely on its mathematical definition without entering in semantical issues which will indeed be touched when describing the different models.

With the term orthopair we intend a pair of orthogonal or disjoint subsets $(A, B)$ of a given universe $\mathrm{X}: A, B \in X$ and $A \cap B=\emptyset$. We will denote the collection of all orthopairs on $X$ as $\mathcal{O}(X):=\{(A, B): A, B \subseteq X, A \cap B=\emptyset\}$. Such pairs have been studied by several authors and in several environments. For instance, Narin'yani in [39] introduced the notion of sub-definite set or in the rough sets field they are investigated in [16] under the name classical preclusivity propositions. In an independent way, Çoker [22] studied them under the name intuitionistic set, the name being due to the fact that they are seen as a classical weakening of so-called Intuitionistic Fuzzy Sets $[2,1]$. Then, following Çoker work, they were renamed double sets in [24].

Further, an orthopair $(A, B)$ is equivalent to a pair of nested sets $(A, C)$ such that $\left(A \subseteq C\right.$ ), through the mapping $C:=B^{c}$. Pairs of this kind have been introduced by Gentilhomme in [32] with the name flou sets and their algebraic structure has been analysed in [35], within a rough sets framework. They are also studied in [57]

APPEARED IN FUNDAMENTA INFORMATICAE, 108, 287-304, 2011 
under the name of interval sets. The collection of all nested pairs on $X$ will be denoted as $\mathcal{N}(X):=\{(A, C): A, C \subseteq X, A \subseteq C\}$.

Well known models which satisfy the disjoint $A \cap B=\emptyset$ or subsethood $A \subseteq$ $B$ condition are twofold sets [26], shadowed sets $[44,45]$, interval sets $[52,56]$, conditional events $[27,51]$ and any rough set based on a Boolean algebra [43]. As a first step of the present work, we review all these models of orthopairs (this is a complete list, of course to the best of our knowledge), putting in evidence the peculiarity of each of them. Connections with three-valued sets and bipolar information [28] will also be highlighted.

Then, we turn our attention to mathematical aspects, in particular to operations and algebraic structures. Due to a bijection between orthopairs and three-valued sets, we can derive properties on orthopairs from the equivalent ones in the threevalued situation. In particular, we can study operations and algebraic structures on three values and then report the results on orthopairs. The study on operations (and hence on algebraic structures) becomes more problematic when we consider subsets of all the orthopairs $\mathcal{O}(X)$, as it happens in some models, in particular in rough sets. Indeed, given an operation, it must be shown that it is closed on that particular subset. This is not simple and even if from a theoretical point of view successful, it can raise questions on the interpretation. This topic will be discussed in the rough sets case, showing with examples where the problem lies.

\section{ORTHOPAIRS MODELS}

The aim of this section is to introduce and review different models of the orthopair concept, showing their similarity and differences both from a mathematical and a semantical point of view. First of all, for a given orthopair $(A, B)$ let us define the two further sets $U:=B^{c}$ and $B n d:=X \backslash(A \cup B)$. These two sets will play a role in several of the following models and their name is due to the meaning they will assume: $U$ stands for Upper and $B n d$ for Boundary, whenever $A$ is interpreted as Lower and $B$ as Exterior of some set under approximation.

2.1. Orthopairs and three valued sets. As a first step, let us make evident the relationship between orthopairs and three-valued sets. Mathematically, an orthopair is in bijection with a three-valued set. Indeed, given a pair $(A, B)$ of disjoint sets on $X$, we can define a three-valued set $f: X \mapsto\left\{0, \frac{1}{2}, 1\right\}$ as

$$
f(x)= \begin{cases}1 & \text { if } x \in A \\ 0 & \text { if } x \in B \\ \frac{1}{2} & \text { ortherwise }\end{cases}
$$

Vice versa, given a three-valued set we can obtain in an obvious way an orthopair $(A, B): x \in A$ if $f(x)=1$ and $x \in B$ if $f(x)=0$.

Thus, we have an isomorphism between the collection of three-valued sets $\mathcal{F}_{\frac{1}{2}}(X):=$ $\left\{f \mid f: X \mapsto\left\{0, \frac{1}{2}, 1\right\}\right\}$ and the collection of disjoint subsets of $X$, i.e., $\mathcal{O}(X)$.

This connection will be useful in the following two ways. At first, it can be used to link three-valued models to rough sets. Then, it enables us to study operations and algebraic structure on $\mathcal{F} \frac{1}{2}(X)$ and report them on orthopairs on $X$. This can be very useful since the study on three values is easier and it is a well known field of research. Let us remark that this link, as expressed by equation 1, has been already introduced in rough sets theory since [41]. 
Remark 1 . The value $\frac{1}{2}$ is the usual value to denote an uncertain situation in threevalued logics, which is half-way between truth (1) and falsity (0). For the safe of simplicity in the mathematical treatment and in compliance with the standard literature, the value $\frac{1}{2}$ is used instead of other numerical values $\frac{1}{3}, \frac{1}{4}, \ldots$ or symbolic ones $a,(0,1), \ldots$ According to the situation/logic it can assume different semantics, such as possible, unknown, undefined, etc. At this point, we do not give a specific interpretation of this value, which can vary from model to model.

2.2. Rough sets. Let us introduce the notions at the basis of rough sets (for an overview and a complete bibliography see [43, 42]). We will first define classical rough sets and then discuss their generalizations.

Definition 2.1. An approximation space is a pair $(X, E)$ with $X$ a set of objects and $E$ an equivalence (reflexive, simmetric, transitive) relation on $X$. Equivalence classes are denoted as $g_{E}(x)$.

An equivalence class is the granule of information associated to an object $x$ and it contains all the objects equivalent to $x$ with respect to $E$. On any approximation space and using these granules, it is possible to define the lower and upper approximations of a given set.

Definition 2.2. Let $(X, E)$ be an approximation space. The lower approximation of $H \subseteq X$ is

$$
l(H):=\left\{x \in X \mid g_{E}(x) \subseteq H\right\}
$$

and the upper approximation of $H$ is

$$
u(H):=\left\{x \in X \mid g_{E}(x) \cap H \neq \emptyset\right\}
$$

A rough set is the pair lower-upper $r(H):=(l(H), u(H))$ or equivalently the pair lower-exterior $r_{e}(H):=(l(H), e(H)):=\left(l(H), u^{c}(H)\right)$.

The lower approximation of a set $H$ contains the objects that certainly belong to $H$ and the upper approximation those that possibly belong to $H$. Thus, $e(H)$ represents the impossibility domain: the objects that certainly do not belong to $H$ and the boundary $\operatorname{Bnd}(H)=u(H) \backslash l(H)$ is the uncertainty region.

As can be seen a rough set is made of a nested pair of sets (equivalently an orthopair). Thus, the collection $\mathcal{R}(X)$ of all rough sets on a given universe $X$ is a subset of $\mathcal{N}(X)$ (or equivalently of $\mathcal{O}(X)$ ). It is worth noticing that in general not all nested pairs can be obtained as approximations of a subset $H$ of the universe $X$, i.e., $\mathcal{R}(X) \varsubsetneqq \mathcal{O}(X)$. This poses some problems when introducing operations of $\mathcal{R}(X)$, since it is not assured that even if the result is a nested (ortho) pair it is also a rough approximation. This topic will be further discussed in Section 3.3. We also remark that besides $(l(H), e(H))$ another orthopair can be defined: $(l(H), B n d(H))$. As a reviewer pointed out, this last notation is handy when the set $H$ is exact (i.e., definable as union of granules) since in this case $B n d(H)=\emptyset$.

Typically, in rough-sets applications, the equivalence relation is obtained looking at the properties of objects: two objects are equivalent if they assume the same value for all the properties under investigation. The structure used to deal with these ideas is known as Information Table or Information System [40].

Definition 2.3. An Information Table is a structure $\mathcal{K}(X)=\langle X, A$, val, $F\rangle$ where:

- the universe $X$ is a non empty set of objects;

- $A$ is a non empty set of attributes; 
D. CIUCCI

- $v a l$ is the set of all possible values that can be observed for an attribute $a$ from $A$ in the case of an object $x$ from $X$;

- $F$ (called the information map) is a mapping $F: X \times A \rightarrow$ val which associates to any pair object $x \in X$ and attribute $a \in A$, the value $F(x, a) \in$ val assumed by $a$ for the object $x$.

Given a set of attributes $D \subseteq A$, two objects $x, y \in X$ are called indiscernible with respect to $D$, and we write $x I_{D} y$, iff $\forall a \in D, F(a, x)=F(a, y)$.

It can be easily verified that $I_{D}$ is an equivalence relation and so for any set of attributes $D$, the pair $\left\langle X, I_{D}\right\rangle$ is an approximation space. Thus, $I_{D}$ partitions the universe $X$ in disjoint classes (granules) $g_{I_{D}}(x)$ defined as $g_{I_{D}}(x):=\{y \in X$ : $\left.x I_{D} y\right\}$.

Finally, we introduce the notion of Rough Membership function which will be useful later. Basically, it is the degree of inclusion of an element $x$ in a set $H$.

Definition 2.4. Given an Information Table $\mathcal{K}(X)$ and a set of attributes $D \subseteq A$, the Rough Membership function of a set $H \subseteq X$ is defined as

$$
\mu_{H}: X \mapsto[0,1] \quad \mu_{H}(y):=\frac{\left|g_{I_{D}}(y) \cap H\right|}{\left|g_{I_{D}}(y)\right|}
$$

Example 2.1. With respect to the Information Table 1, let us consider the indiscernibility relation generated by the set of attributes, $I_{\mathcal{A}}=\{$ Pressure,Headache,Temperature,Muscle Pain $\}$, then the partition of the universe $X$ is made of the following equivalence classes: $\{P 1\},\{P 2, P 3\},\{P 4\},\{P 5\}$.

\begin{tabular}{|c|c|c|c|c|c|}
\hline Patient & Pressure & Headache & Temperature & Muscle Pain & Flu \\
\hline$P 1$ & Normal & yes & $38-39$ & yes & A \\
$P 2$ & High & no & $36-37$ & yes & A \\
$P 3$ & High & no & $36-37$ & yes & B \\
$P 4$ & Low & yes & $35-36$ & no & no \\
$P 5$ & Normal & yes & $36-37$ & yes & no \\
\hline
\end{tabular}

TABLE 1. An example of Information Table

If we consider the set of objects $H=\{P 1, P 2\}$, that is the set of patient having flu $\mathrm{A}$, then its rough approximation is $r(H)=\langle\{P 1\},\{P 1, P 2, P 3\}\rangle$. That is, we are sure that patients with symptoms as $P 1$ have flu $\mathrm{A}$ and $P 4, P 5$ do not have it. However, due to the inconsistency between $P 2$ and $P 3$ (same symptoms but different $\mathrm{flu}$ ), we can only say that someone with symptoms as $P 2$ and $P 3$ could have flu A, but we are not sure about it. This situation is reflected also by the membership function $\mu_{H}$ which is defined as $\mu_{H}(\mathrm{P} 1)=1, \mu_{H}(\mathrm{P} 2)=\mu_{H}(\mathrm{P} 3)=\frac{1}{2}$ and $\mu_{H}(\mathrm{P} 4)=\mu_{H}(\mathrm{P} 5)=0$.

Several generalizations of this classical definition of rough sets have been introduced. They are based on the power set of a given universe or also on a fuzzy or abstract context. To the scope of the present work, only the former are interesting, since we are dealing with pair of sets ${ }^{2}$. In this category we can find approximations defined by means of a general (i.e., not equivalence) binary relation [11, 53, 34, 42], approximations based on covering and neighborhoods [54], variable precision rough

\footnotetext{
${ }^{2}$ Pairs of orthogonal fuzzy sets gives rise to so called intuitionistic fuzzy sets [1]
} 
sets [58, 36], probabilistic rough sets [55]. All these approaches are based on the power set of a universe and satisfy the sub-sethood property (see [17]), thus they lead to different models of orthopairs.

Another light under which we can investigate the relationship between rough sets and orthopairs is outlined in [37]. The authors call orthopairs samples with the idea that they give positive and negative examples about the same unknown concept. With respect to this idea, the relationship between samples (orthopairs) and rough sets is then analyzed. The following two notions are first introduced:

- adequacy. An information system is adequate for an orthopair $(P, N)$ if for no elements $x \in P, y \in N$ it happens that $x, y$ are equivalent (that is $\left.x I_{D} y\right)$.

- consistency. A rough set $(l(A), u(A))$ is consistent with an orthopair $(P, N)$ if $P \subseteq l(A), N \cap u(A)=\emptyset$. An information system is consistent with an orthopair $(P, N)$ if there is a rough set over $I$ consistent with $(P, N)$.

Thus, adequacy implies that the information system is not in contradiction with the observed example, whereas consistency represents the idea that the knowledge carried by the example can be described by a rough set. Interestingly enough, these two notions turn out to be equivalent.

2.3. Twofold sets. Twofold sets [26] were conceived to account for those situations where in front of a lack of knowledge we want to understand which is the set of objects $X_{+}$satisfying a certain property $P$ and which objects $X_{-}$do not satisfy $P$. Clearly, $X_{+} \cap X_{-}=\emptyset$ and they form an orthopair.

More precisely, given a universe $X$, we define a multivalued attribute $a$ with domain val as a mapping $\Gamma_{a}: X \mapsto \mathcal{P}(v a l)$. The set $\Gamma_{a}(x) \subseteq$ val represents the possible values for $a$ of $x$ which due to a lack of knowledge is not exactly known.

Let $V \subseteq$ val be a subset of values. The ill-known set $\Gamma_{a}^{-1}(V) \subseteq X$ is approximated by the twofold set $\left\langle\Gamma_{a *}^{-1}(V), \Gamma_{a}^{-1 *}(V)\right\rangle$ where

$$
\begin{aligned}
\Gamma_{a *}^{-1}(V) & :=\left\{x \in X, \Gamma_{a}(x) \subseteq V\right\} \\
\Gamma_{a}^{-1 *}(V) & :=\left\{x \in X, \Gamma_{a}(x) \cap V \neq \emptyset\right\}
\end{aligned}
$$

Thus, the set of objects which share the same value for the attribute $a$ is approximated by the lower and upper bounds $\left\langle\Gamma_{a *}^{-1}(V), \Gamma_{a}^{-1 *}(V)\right\rangle$ which is a pair of nested sets.

Example 2.2. Let us modify the attribute Pressure of Table 1 according to Table 2 , where due to a lack of knowledge we are not sure about the data of patients $P 1$ and $P 4$.

\begin{tabular}{|c|c|c|c|c|c|}
\hline Patient & Pressure & Headache & Temperature & Muscle Pain & Flu \\
\hline$P 1$ & $\{$ Normal,High $\}$ & yes & $38-39$ & yes & $\mathrm{A}$ \\
$P 2$ & High & no & $36-37$ & yes & A \\
$P 3$ & High & no & $36-37$ & yes & B \\
$P 4$ & $\{$ Low,Normal $\}$ & yes & $35-36$ & no & no \\
$P 5$ & Normal & yes & $36-37$ & yes & no \\
\hline
\end{tabular}

Now, we ask which are the patients with a normal pressure, i.e., $V=\{$ Normal $\}$. Using the above definitions we get $\Gamma_{\text {Pressure* }}^{-1}(\{$ Normal $\})=\{P 5\}$ and $\Gamma_{\text {Pressure }}^{-1 *}(\{$ Normal $\})=$ 
$\{P 1, P 4, P 5\}$. That is, for sure P5 has normal pressure and P2,P3 do not have normal pressure, whereas we cannot be certain about patients $\mathrm{P} 1$ and $\mathrm{P} 4$.

When comparing rough and twofold sets we get that from a theoretical standpoint they are similar. Indeed, formally, if in equations (2) we change $\Gamma_{a}(x)$ with $g_{E}(x)$ and attributes with objects (that is, $D$ with $X$ ), we get exactly the lower and upper approximations of definition 2.2. Let us note that also in the two-fold case, not all nested pairs can be retrieved as approximations of some property. That is, the collection $R_{t f}(X)=\left\{\left\langle\Gamma_{a *}^{-1}(V), \Gamma_{a}^{-1 *}(V)\right\rangle: a \in A t t, V \subseteq v a l\right\}$ is a subset of $\mathcal{N}(X)$. For instance, in the above example 2.2 , the pair $(\{P 5\},\{P 4, P 5\})$ cannot be obtained by any attribute $a$ and any set of values $V$.

On the contrary, when considering the interpretation of the two approaches, they express two different points of view. In both cases we have the same type of vagueness [30] where the objects are partitioned in two crisp sets, but the cause of uncertainty is different. In twofold sets we have a precise set of attributes $(F)$ which however is not able to precisely characterize a set of objects. In rough sets we have a well-known set of objects $(H)$ which cannot be precisely characterized by the attributes. In some sense, with twofold sets we know the intension of the set under investigation but not the extension and vice versa in rough sets we now the extension but not the intension.

2.4. Shadowed sets. Fuzzy sets are a powerful tool to describe and reason with vague concepts, indeed they permit to express vagueness with an infinite degree of accuracy, i.e., any value in the range $[0,1]$. However, this quality in precision may not always be needed or even worst may lead to high computational costs. Besides the well known level fuzzy sets and $\alpha$-cuts, more recently Pedrycz introduced shadowed sets $[44,45,47]$ as a new tool to approximate a fuzzy set and thus to deal with vagueness in a simpler way. According to him, the intention was "to introduce a model which does not lend itself to precise numerical membership values but relies on basic concepts of truth values (yes - no) and on entire unit interval perceived as a zone of uncertainty" [44]. Formally, a shadowed set can be defined as follows.

Definition 2.5. Let $X$ be a set of objects, called the universe. A shadowed set on $X$ is any mapping $s: X \rightarrow\{0,1,(0,1)\}$.

From any fuzzy set it is possible to obtain a corresponding shadowed set. In order to define such a mapping, it is sufficient to fix a value $\alpha \in\left[0, \frac{1}{2}\right)$. Then, for a given fuzzy set $f$, the membership values $f(x)$ which are less than or equal to $\alpha$ are set to 0 and those greater than or equal to $(1-\alpha)$ are set to 1 . The remaining ones, i.e., the membership values belonging to $(\alpha, 1-\alpha)$, are set to $(0,1)$, since they are characterized by a great uncertainty or lack of knowledge and they are consequently considered the "shadow" of the induced shadowed set.

Clearly, a shadowed set reduces a fuzzy set to a three-valued set and hence to an orthopair (see Section 2.1). With this interpretation an orthopair represents the certainty we have on a given fuzzy set.

From a formal point of view we can also recover a shadowed set from a rough set. Indeed, let $\mu_{H}$ be the membership function of set $H$. The $\alpha$-shadowed set 
generated by $\mu_{H}$ is defined as

$$
\begin{cases}0 & \text { if } \mu_{H}(y) \leq \alpha \\ 1 & \text { if } \mu_{H}(y) \geq 1-\alpha \\ (0,1) & \text { otherwise }\end{cases}
$$

Example 2.3. Let us consider the rough membership function defined in example 2.1 and set $\alpha=0.3$. The induced shadowed set is $s(P 1)=1, s(P 2)=s(P 3)=(0,1)$ and $s(P 4)=s(P 5)=0$.

However, from a semantic point of view, shadowed and rough sets have different foundations and different motivations. Further, in the rough-sets case "the approximation space is defined in advance and the equivalence classes are kept fixed" whereas in shadowed sets the three regions are assigned dynamically by selecting a proper $\alpha^{4}$. In this sense we note that a peculiarity of shadowed set with respect to other ways to approximate a fuzzy set $[12,18]$ is that they give the way to select the "best" $\alpha$ according with a Balance of Vagueness criterion [47]: "the primary goal we intend to accomplish in this way is the one of localizing and balancing the factor of uncertainty that is inherently associated with any fuzzy set". Indeed, a shadowed set localizes uncertainty by moving it from the intervals $[0, \alpha)$ and $(1-\alpha, 1]$ to the interval $(\alpha, 1-\alpha)$, i.e., to the "shadow". In order to balance this exchange of information, Pedrycz proposes the following method.

For a given fuzzy set $f: X \mapsto[0,1]$ and for every $\alpha \in(0,1 / 2]$, let us divide the universe $X$ in three regions:

$$
\begin{aligned}
& \Omega_{1}(\alpha)=\{x: f(x) \in[0, \alpha]\} \\
& \Omega_{2}(\alpha)=\{x: f(x) \in(1-\alpha, 1]\} \\
& \Omega_{3}(\alpha)=\{x: f(x) \in[\alpha, 1-\alpha)\}
\end{aligned}
$$

Then, the optimal $\alpha$ is the one minimizing (if possible, setting to zero) the following quantity $[47,46]$ :

$$
V_{f}(\alpha):=\left|\int_{\Omega_{1}(\alpha)} f(x) d x+\int_{\Omega_{2}(\alpha)}(1-f(x)) d x-\int_{\Omega_{3}(\alpha)} d x\right|
$$

The possibility to compare different values of $\alpha$ and choosing the best one is clearly useful in applications.

2.5. Interval sets. An interval set is formally defined as a pair of nested sets, that is given a universe $X$ the collection of interval sets is exactly $\mathcal{N}(X)$. They were first introduced in [52] and for a recent overview see [56].

The peculiarity of interval sets is in the semantic attached to them. Indeed, an interval set $(L, U)$ is interpreted as a "family of sets", lower bounded by $A$ and upper bounded by $U$. This semantic is useful when dealing with partially known concepts. If the only available knowledge about a concept $C$ is that $C \in(L, U)$ we only know that $L \subseteq C \subseteq U$, that is, $C$ is a set in the family of all sets between $L$ and $U$.

The reason of this partial knowledge in not fixed a priori, it can derive from a lack of information in intension or in extension. As a consequence, different operations inspired by different semantics can be introduced on interval sets [56].

\footnotetext{
${ }^{4}$ This is no more valid in some rough-sets model, for instance VPRS [58, 36] and probabilistic [55], where the lower and upper approximations are explicitly defined using one or more parameters $[12,17]$
} 
Example 2.4. On the universe $X=\{P 1, P 2, P 3, P 4, P 5\}$ an interval set is $(\{P 1\},\{P 1, P 2, P 3\})$ which represents a set $C$ in the family $\{\{P 1\},\{P 1, P 2\},\{P 1, P 2, P 3\}\}$ but we are not sure which of them. Keeping in mind the semantic given in example 2.1 and interpreting the set $C$ as having flu $A$, it is clear that $C$ is only partially known: for sure $P 1 \in C$, but nothing can be said about $P 2$ and $P 3$. Clearly, according to this interpretation, the lack of knowledge is given by the attributes, that is in intension.

2.6. Conditional events. A conditional event (or conditional object) is an attempt to formalize the relationship between two events (or between two logical propositions) when one depends on the other.

Often a three-valued semantics has been used for conditional events where besides true and false, the third value means undefined, that is with the available knowledge it is not possible or it does not make sense to say if a conditional event is true or false.

As stated in [27] this leads to an interval and thus to an orthopair: "a conditional object can be equated to a pair of nested sets", where the lower (resp., upper) bound is obtained by interpreting the undefined value as false (resp., true). Thus, as in the interval set case, we can think to a conditional object as a family of sets where only one on them corresponds to the exact extension of the object under investigation, but due to a lack of knowledge we do not know it exactly.

Example 2.5. Let us consider the two events $E 1=$ "It is raining" and $E 2=$ "Open the umbrella". Then the conditional event $E 2 \mid E 1$ is true if both $E 1$ and $E 2$ are true, false if $E 1$ is true and $E 2$ is false, undefined if $E 1$ is false. As can be seen the conditional event can be interpreted as a rule "if it is raining then open the umbrella" and as such it can be meaningful in rule-based systems. According to this interpretation, it is clear that if $E 1$ is false (i.e., it is not raining) the rule is not applicable, hence, the value of the whole rule is undefined. Further, according to the transformation of a three valued set into an orthopair $(A, B)$, we can interpret $A$ as the collection of all examples of the rule, i.e., $A=E 1 \cap E 2$, and $B$ as the set of counterexamples, $B=E 1 \cap E 2^{c}$.

From a formal standpoint, several representations can be given of conditional events. For instance Walker [51] gives six (equivalent) of them, and in particular the number 5 is exactly the definition of an orthopair on Boolean algebras.

Definition 2.6. Let $\left(A, \wedge, \vee,{ }^{\prime}, 0,1\right)$ be a Boolean algebra. Then the set of conditional events on $A$ is

$$
\mathcal{C}(A):=\{(a, b): a, b \in A, a \wedge b=0\}
$$

2.7. Bipolar information. Orthopairs can be seen as a way to represent bipolar information. Indeed, "Bipolarity refers to the propensity of the human mind to reason and make decisions on the basis of positive and negative affects" [28]. Typical examples of bipolar information are pros and cons of a decision or examples and counterexamples of the same statement. Conditional events viewed as decision rules represent this situation, where, as laid bare in example 2.5 , we can build an orthopair from a rule, by considering its examples and counterexamples.

There are several types of bipolar information depending on the data used to represent knowledge and if positive and negative information is given by the same 
agent or not [28]. Orthopair models are usually of type II: Symmetric bivariate unipolarity. That is, given an orthopair $(A, B)$ :

- $A, B$ are defined on the basis of the same data

- $A, B$ are defined by two independent judgements on unipolar scales

Rough sets belong to this category when the pair (lower, exterior) is considered and the notion of sample introduced in section 2.2 clearly outlines the bipolar aspect of rough sets. Also conditional rules and interval sets belong to this category where the "two independent judgments" are respectively given by examples and counterexample [28] and by the instrument used to define the lower and upper bound of the interval, which can vary from situation to situation.

On the contrary three-valued sets are of type I, Symmetric univariate bipolarity:

- single evaluation on a bipolar scale: false, half-true, true.

Hence, also shadowed sets belong to this category.

Moreover, orthopairs can arise also in Asymmetric bipolarity (Type III). In this case the two sets of an orthopair are generated on different data, hence the asymmetry. For instance, background knowledge from one side (negative information) and empirical data (positive information) from the other.

Let us note that if we report this kind of bipolarity on rough sets, it is possible to think to a new rough sets model where the lower and upper approximations are defined on different data. Of course in this case the relationship between lower and upper approximations are not standard and it should be accurately investigated.

As a conclusion we summarize in Table 2.7 the characteristic of the models analyzed in the present section.

\section{Operation ON ORTHOPAIRS}

As stated in section 2.1 thanks to the bijection between orthopairs and threevalued sets it is possible to study operations and algebraic structures on three values and then report them on orthopairs. So, for instance, we have that only two binary operations corresponding to a discrete t-norm can be defined on orthopairs [6]. Similarly, we can have 14 implications $[19,20]$ and only two are a residual of a t-norm, namely, the Lukasiewicz and Gödel ones. Particular attention should be paid when reporting these results on orthopair models, since we must assure that the operations are well defined on that particular model: in subsection 3.3 we will briefly discuss the rough set case. In the following we are not going to study all such operations, instead we refer to $[21,19,20]$. Here, we give a brief overview of some known results on the algebraic approach and we will focus on lattice operations and related order relations.

3.1. Lattice operations and order relations. The usual order relation on threevalued (fuzzy) sets is the point-wise one:

$$
f_{1} \leq_{1} f_{2} \quad \text { iff } \quad \forall x f_{1}(x) \leq f_{2}(x) \quad \text { where } \quad 0 \leq \frac{1}{2} \leq 1
$$

That is, the usual number ordering on $\left\{0, \frac{1}{2}, 1\right\}$ is extended to fuzzy sets. If we consider the equivalent order on orthopairs we get

$$
\left(A_{1}, B_{1}\right) \sqsubseteq_{1}\left(A_{2}, B_{2}\right) \quad \text { iff } \quad A_{1} \subseteq A_{2}, B_{2} \subseteq B_{1}
$$




\begin{tabular}{l|l|l|l} 
Model & Type & Bipolarity & Comment \\
\hline Rough Sets & $\begin{array}{l}\mathcal{R}(X) \subseteq N(X) \\
R_{e}(X) \subseteq \mathcal{O}(X)\end{array}$ & II & $\begin{array}{l}\text { Approximation of sets } \\
\text { with precise extension } \\
\text { and ill-known intension }\end{array}$ \\
\hline Twofold Sets & $R_{t f} \subseteq \mathcal{N}(X)$ & II & $\begin{array}{l}\text { Approximation of sets } \\
\text { with precise intension } \\
\text { and ill-known extension }\end{array}$ \\
\hline Shadowed Sets & $\mathcal{O}(X)$ & I & $\begin{array}{l}\text { Approximation of fuzzy } \\
\text { sets; regions dynami- } \\
\text { cally defined through a } \\
\text { parameter } \text { a differently } \\
\text { from classical rough sets } \\
\text { and similarly to VPRS; } \\
\text { a criterion to define the } \\
\text { best } \alpha \text { is given }\end{array}$ \\
\hline Interval Sets & $\mathcal{N}(X)$ & & $\begin{array}{l}\text { A family of sets used to } \\
\text { represent an ill-known } \\
\text { set in extension or in in- } \\
\text { tension }\end{array}$ \\
\hline Conditional Events & $\mathcal{N}(X), \mathcal{O}(X)$ & II & $\begin{array}{l}\text { It does not give an ap- } \\
\text { proximation of a set but } \\
\text { the relationship between } \\
\text { two events; useful in } \\
\text { representing conditional } \\
\text { rules }\end{array}$ \\
& & & II
\end{tabular}

TABLE 3. Overview of models features

This is indeed the most used order relation on orthopair models. It leads also to a lattice structure where the meet and join are defined respectively as

$$
\begin{array}{ll}
\left(A_{1}, B_{1}\right) \sqcap_{1}\left(A_{2}, B_{2}\right) & :=\left(A_{1} \cap A_{2}, B_{1} \cup B_{2}\right) \\
\left(A_{1}, B_{1}\right) \sqcup_{1}\left(A_{2}, B_{2}\right) & :=\left(A_{1} \cup A_{2}, B_{1} \cap B_{2}\right)
\end{array}
$$

We note that in $[37,56]$ this ordering is named inclusion ordering. Indeed, if the semantic of an orthopair is to approximate a not exactly known set, this order relation means that an orthopair $\left(A_{1}, A_{0}\right)$ is less than another one $\left(B_{1}, B_{0}\right)$ if they are approximations of two sets $A, B$ such that $A \subseteq B$. Further, in the context of conditional events the conjunction (7) is named interval conjunction since it "is in complete accordance with the interval understanding of conditional objects" (see [27] for more details).

In [37] another order relation on orthopairs is introduced and named sub-sethood:

$$
\left(A_{1}, B_{1}\right) \sqsubseteq_{2}\left(A_{2}, B_{2}\right) \quad \text { iff } \quad A_{2} \subseteq A_{1}, B_{2} \subseteq B_{1}
$$

This relation, once interpreted on three values does not generate a lattice but only the join-semilattice of figure 1 . 


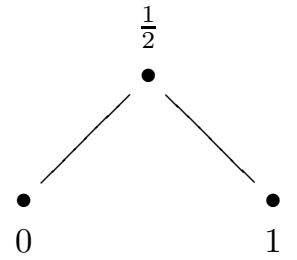

Figure 1. The lattice structure of order 3.

Clearly, this happens also on orthopairs where the meet does not always exist and the join is defined as:

$$
\left(A_{1}, B_{1}\right) \sqcup_{2}\left(A_{2}, B_{2}\right):=\left(A_{1} \cap A_{2}, B_{1} \cap B_{2}\right)
$$

By relaxing the requirements of this order we can obtain two other orderings, which generate two lattice operations. In one case we keep the condition on the first component and in the other the condition on the second one. That is, the new order relations on orthopairs are:

$$
\begin{aligned}
& \left(A_{1}, B_{1}\right) \sqsubseteq_{3}\left(A_{2}, B_{2}\right) \quad \text { iff } \quad B n d_{1} \subseteq B n d_{2}, B_{2} \subseteq B_{1} \\
& \left(A_{1}, B_{1}\right) \sqsubseteq_{4}\left(A_{2}, B_{2}\right) \quad \text { iff } \quad A_{2} \subseteq A_{1}, B n d_{1} \subseteq B n d_{2},
\end{aligned}
$$

where the condition $B n d_{1} \subseteq B n d_{2}$ can be equivalently expressed as $A_{2} \cup B_{2} \subseteq$ $A_{1} \cup B_{1}$.

It can be easily seen that order 2 implies orders 3 and 4 :

$$
\text { If }\left(A_{1}, B_{1}\right) \sqsubseteq_{2}\left(A_{2}, B_{2}\right) \text { then }\left(A_{1}, B_{1}\right) \sqsubseteq_{3}\left(A_{2}, B_{2}\right),\left(A_{1}, B_{1}\right) \sqsubseteq_{4}\left(A_{2}, B_{2}\right)
$$

but not vice versa. Indeed, both orders 3 and 4 must be satisfied to recover order 2:

If $\left(A_{1}, B_{1}\right) \sqsubseteq_{3}\left(A_{2}, B_{2}\right) \quad$ and $\quad\left(A_{1}, B_{1}\right) \sqsubseteq_{4}\left(A_{2}, B_{2}\right) \quad$ then $\left(A_{1}, B_{1}\right) \sqsubseteq_{2}\left(A_{2}, B_{2}\right)$

On three values orders (11) and (12) correspond to a different order with respect to the standard one on numbers, according to the following equations:

$$
\begin{aligned}
& f_{1} \leq_{3} f_{2} \quad \text { iff } \quad \forall x f_{1}(x) \leq f_{2}(x) \quad \text { where } \quad 0 \leq 1 \leq \frac{1}{2} \\
& f_{1} \leq_{4} f_{2} \quad \text { iff } \quad \forall x f_{1}(x) \leq f_{2}(x) \quad \text { where } \quad 1 \leq 0 \leq \frac{1}{2}
\end{aligned}
$$

So, in some sense, $0, \frac{1}{2}, 1$ are no more treated as numbers with associated order, but as labels with an order. The analysis of the semantic of these orders and the following logics are out of the scope of the present work. Instead, we are going to see that they have an intuitive interpretation on orthopairs.

These two orderings give rise to the following meet and join operations on orthopairs

$$
\begin{array}{ll}
\left(A_{1}, B_{1}\right) \sqcap_{3}\left(A_{2}, B_{2}\right) & :=\left(A_{1} \backslash B_{2} \cup A_{2} \backslash B_{1}, B_{1} \cup B_{2}\right) \\
\left(A_{1}, B_{1}\right) \sqcup_{3}\left(A_{2}, B_{2}\right) & :=\left(A_{1} \backslash B n d_{2} \cup A_{2} \backslash B n d_{1}, B_{1} \cap B_{2}\right) \\
\left(A_{1}, B_{1}\right) \sqcap_{4}\left(A_{2}, B_{2}\right) & :=\left(A_{1} \cup A_{2}, B_{1} \backslash A_{2} \cup B_{2} \backslash A_{1}\right) \\
\left(A_{1}, B_{1}\right) \sqcup_{4}\left(A_{2}, B_{2}\right) & :=\left(A_{1} \cap A_{2}, B_{1} \backslash B n d_{2} \cup B_{2} \backslash B n d_{1}\right)
\end{array}
$$

We note that in the conditional event context the operations (15) and (16) are named quasi-conjunction and quasi-disjunction and together with operations (7) 
and (8) are the only two possibilities which satisfy the constraints derived by their use as inference rules [27].

From a semantic standpoint, we have a more clear picture if we invert the orders, that is: $\left(A_{2}, B_{2}\right) \preceq_{i}\left(A_{1}, B_{1}\right)$ iff $\left(A_{1}, B_{1}\right) \sqsubseteq_{i}\left(A_{2}, B_{2}\right)$ with $i \in\{2,3,4\}$. We note that $\preceq_{2}$ is called knowledge ordering in [56] and it represents the idea that we have more knowledge about $\left(A_{2}, B_{2}\right)$ than about $\left(A_{1}, B_{1}\right)$. A similar thing can be said about orderings $\preceq_{3}$ and $\preceq_{4}$. Indeed, the fact that with $\preceq_{3}$ and $\preceq_{4}$, we have $B n d_{1} \subseteq B n d_{2}$, means that we are more certain in situation 1 than in situation 2 . The following pictures represent these three orderings.

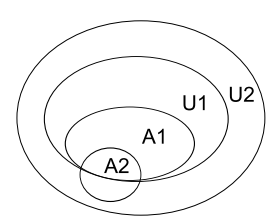

(a)

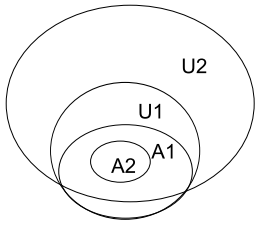

(b)

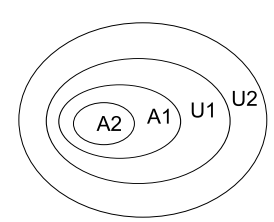

(c)

FiguRE 2. Representations of orders $\preceq_{3}, \preceq_{4}$ and $\preceq_{2}$.

Thus, orderings 3 and 4 are less demanding from the "knowledge" point of view of ordering 4 , but they have the advantage to generate a lattice structure, with the possibility to define intersection and union. We note however, that the operations generated by order three are quite "strange". Indeed, due to the fact that the natural order between 0 and 1 is inverted, we have the counter-intuitive behaviour that with the intersection (union), the first component of the result is obtained by the union (intersection) of the two operands.

More study about the role that these orderings can play in the different applications is of course needed. Also some investigations about the structures arising from them would be interesting. For instance, in [37] is it said that orders $\sqsubseteq_{1}$ and $\preceq_{2}$ forms a bilattice on the collection of all (not necessarily ortho) pairs of sets. Clearly, this is not true on $\mathcal{O}(X)$ since order $\preceq_{2}$ does not give rise to a lattice.

3.2. Algebraic structure. The collection of ortho (nested) pairs can be endowed with different operations (some have been introduced in the previous subsection) and thus algebraic structures. Here, we give some hints about some known results on this topic.

Iwinski [35] studied the collection of orthopairs in connection with rough sets ideas and showed that $\left(\mathcal{N}(X), \sqcap_{1}, \sqcup_{1},(\emptyset, X),(X, \emptyset)\right.$ is a distributive lattice. This result can be improved in different ways:

- The structure $\left\langle\mathcal{N}(X), \sqcap_{1}, \sqcup_{1}, \approx,(\emptyset, X),(X, \emptyset)\right\rangle$ is a Stone algebra [49] where $\approx$ is defined as

$$
\approx(A, B):=\left(B, B^{c}\right)
$$

- The structure $\left\langle\mathcal{N}(X), \sqcap_{1}, \sqcup_{1}, \approx,-,(\emptyset, X),(X, \emptyset)\right\rangle$ is a BZ lattice [16] where

$$
-(A, B):=(B, A)
$$

Moreover, in [10] the structure of orthopairs $\mathcal{O}(X)$ is related to the notion of texture space. In particular, texture spaces are characterized in order to be "intuitionistic textures", i.e., texture isomorphic to the collection of orthopairs $\mathcal{O}(X)$. 
Further, several structures are studied in connection with rough sets and can be thus easily introduced also on orthopairs. For an overview we refer to [4]. Here, we just mention that the richer structure used in connection with rough sets is the one of Heyting Wajsberg algebra $[13,14]\left\langle\mathcal{R}(X), \Rightarrow_{L}, \Rightarrow_{G},(\emptyset, X)\right\rangle$ where $\Rightarrow_{L}, \Rightarrow_{G}$ are respectively a Łukasiewicz and a Gödel implication. Thus, both Stone algebras and BZ lattices are a substructure of $\mathrm{HW}$ algebras. We also note that $\mathrm{HW}$ algebras are equivalent to other well know structures, without entering into details (see [14]) we mention: $\mathrm{MV}_{\Delta}$ algebras, obtained adding a Baaz's operator $\Delta[3,33]$ to $\mathrm{MV}$ algebras; Stonean MV algebra, a particular class of MV algebras introduced by Belluce [7]; BZMV ${ }^{d M}$ algebras, a pasting of MV algebras and BZ lattices [15].

Moving to a more abstract context, it is possible to define orthopairs on more general spaces than the powerset of a given universe and then study their algebraic structure.

- By defining orthopairs on a Boolean algebra as in definition 2.6, Walker [51] proved that the structure $\langle\mathcal{C}(A), \curlyvee, \curlywedge, \sim,(0,1)\rangle$ is a Stone algebra, where $\curlyvee, \curlywedge$ are the abstract meet and join corresponding to the concrete ones $\sqcap 1, \sqcup_{1}$ :

$$
\begin{aligned}
& (a, b) \curlyvee(c, d):=(a \wedge b, c \vee d) \\
& (a, b) \curlywedge(c, d):=(a \vee b, c \wedge d)
\end{aligned}
$$

$(0,1)$ is the minimum of the lattice, $\sim$ is the corresponding of $\approx$ :

$$
\sim(a, b):=\left(b, b^{\prime}\right)
$$

Moreover, he characterizes those Stone algebras $S$ that are an algebra of orthopairs $\mathcal{O}(A)$ through a condition on the skeleton and dense subsets of $S$.

- The structure of nested pairs of a Boolean algebra, i.e., pairs $(a, b)$ such that $a \leq b$, was studied also by Monteiro [38, p.199]. Translating his results to orthopairs, he showed that $\langle\mathcal{C}(A), \curlyvee, \curlywedge, \neg, \nabla,(1,0)\rangle$ where $\neg(a, b):=(b, a)$ and $\nabla(a, b)=\left(b^{\prime}, b\right)$ is a three-valued

Łukasiewicz algebra.

- Going more generally, and considering an Heyting algebra $\langle A, \wedge, \vee, \rightarrow, 0,1\rangle$, instead of a Boolean one, Vakarelov [50] showed that $\langle\mathcal{C}(A), \curlyvee, \curlywedge, \rightarrow, \neg, \nabla,(1,0)\rangle$ where $(a, b) \rightarrow(c, d):=(a \rightarrow c, a \cap d)$ is a Nelson algebra. The interesting aspect, with respect to the interpretation side, is that this approach has been developed in order to give a semantics to the notion of counterexample, hence it is strictly related to orthopairs and bipolar information.

3.3. Going down to models: the rough set case. All the previous operations (and consequently algebraic structures) regard the collection of all orthopairs $\mathcal{O}(X)$ (nested pairs $\mathcal{N}(X)$ ) of a given universe. If we want to report these results on the models we must pay attention to the fact that they remain closed operators and then understand which is the semantic in that model. More clearly, let us consider a n-ary function $f: \mathcal{O}(X)^{n} \rightarrow \mathcal{O}(X)$ on orthopairs and then consider a subset $S \subseteq \mathcal{O}(X)$ of orthopairs. The problem is if for all $\underline{s} \in S^{n}$ it happens that $f(\underline{s}) \in S$. This is not obvious and sometimes hard to prove. Moreover, if from a theoretical point of view one is able to prove it, the second question is if it makes sense from a semantical standpoint. To show this problem, we now consider the case of rough 
sets, i.e. $S=\mathcal{R}_{e}(X)$, and in particular the usual meet and join operations $\Pi_{1}, \sqcup_{1}$. Let us start with an example.

Example 3.1. Let our universe be $X=\{a, b, c, d, e, f\}$ and suppose to have the following two partitions:

$$
\begin{aligned}
& \pi^{1}=\{a, b\},\{c, d\},\{e, f\} \\
& \pi^{2}=\{a, b, c\},\{d, e, f\}
\end{aligned}
$$

Now, consider the two sets $X_{1}=\{a, b, c\}$ and $X_{2}=\{c, e\}$. We compute their (lowerexterior) approximations with respect to the two partitions and according to definition 2.2. Considering the first partition we have: $r_{e}^{1}(\{a, b, c\})=(\{a, b\},\{e, f\})$, $r_{e}^{1}(\{c, e\})=(\emptyset,\{a, b\})$. Their intersection and union using equations $(7),(8)$ are:

$$
\begin{aligned}
& r_{e}^{1}(\{a, b, c\}) \sqcap_{1} r_{e}^{1}(\{c, e\})=(\emptyset,\{a, b, e, f\}) \\
& r_{e}^{1}(\{a, b, c\}) \sqcup_{1} r_{e}^{1}(\{c, e\})=(\{a, b\}, \emptyset)
\end{aligned}
$$

The question, now, is if there exist two sets $M_{1}, J_{1}$ such that $r_{e}\left(M_{1}\right)=(\emptyset,\{a, b, e, f\})$ and $r_{e}\left(J_{1}\right)=(\{a, b\}, \emptyset)$. The answer is positive but not unique. For instance, $M_{1}=\{c\}$ or $M_{1}=\{d\}$ and $J_{1}=\{a, b, c, e\}$ or $J_{1}=\{a, b, d, f\}$.

With respect to the second partition, we obtain $r_{e}^{2}(\{a, b, c\})=(\{a, b, c\},\{d, e, f\})$, $r_{e}^{2}(\{c, e\})=(\emptyset, \emptyset)$ and then

$$
\begin{aligned}
& r_{e}^{2}(\{b, c\}) \sqcap_{1} r_{e}^{2}(\{c, e\})=(\emptyset,\{d, e, f\}) \\
& r_{e}^{2}(\{b, c\}) \sqcup_{1} r_{e}^{2}(\{c, e\})=(\{a, b, c\}, \emptyset)
\end{aligned}
$$

Also in this case, there are several sets $M_{2}, J_{2}$ which have as rough approximation $(\emptyset,\{d, e, f\})=r_{e}\left(M_{2}\right)$ and $(\{a, b, c\}, \emptyset)=r_{e}\left(M_{2}\right): M_{2}=\{x\}$ with $x \in\{a, b, c\}$ and $J_{2}=\{a, b, c, y\}$ with $y \in\{d, e, f\}$. Moreover, when comparing the solution of the two partitions, it can be easily seen that they are quite different.

In the above example, the two operations $\sqcap_{1}, \sqcup_{1}$ are closed on the collection of all classical rough sets. This is true in general, indeed it was shown how to construct the two sets $M$ and $J$ in several manners $[8,31,5]$. However, as shown in the example, this solution has some problem from the point of view of the interpretation. Indeed,

- they depend on the partition and thus on the available data which can vary during time or from one observer to another;

- also inside the same partition, they are not unique, that is they cannot be exactly described using only the two operands under investigation.

This can be interpreted as a language related issue. The starting sets $X_{1}, X_{2}$ are known in extension (i.e., we known the objects belonging to them) whereas their intension is available only through approximations and the attributes to describe them. Clearly, intension depends on the language and in this case through attributes: the more attributes the finer the description. It is evident that changing the attributes (the language) will change intersection and union but not $X_{1}$ and $X_{2}$. For more discussion about this topic see also [25, 21].

Further, if from classical rough sets, we move to more general approaches, for instance using a tolerance instead of an equivalence relation, we are not even assured on the closeness of the operations [9]. 


\section{Conclusion}

Orthopairs and nested pairs of sets are a widely used notion to represent uncertainty. We have seen that the interpretation as well as the way to obtain them changes from model to model. Several operations can be introduced on the collection of orthopairs on a given universe, giving rise to different algebraic structures. Here, we analyzed some of them, mainly related to order relations and gave an overview of existing algebras used in connection with orthopairs.

Several generalizations can also be studied by abstracting the underlying structure, the only condition is to be able to define an orthogonality relation. So, if we consider fuzzy sets instead of Boolean ones, we get so-called Intuitionistic Fuzzy Sets (and equivalent structures [23]). On the other hand, we can consider pairs on an abstract algebra (Boolean, Heyting, ....) as outlined in Section 3.2.

The bijection between orthopairs and three-valued sets will be further explored in order to study new operations on orthopairs and their models [19].

\section{REFERENCES}

[1] Atanassov, K. T.: Intuitionistic Fuzzy Sets, vol. 35 of Physica Verlag, Springer, Heidelberg, 1999.

[2] Atanassov, K. T., Stoeva, S.: Intuitionistic fuzzy sets, Polish Symp. on Interval \& Fuzzy Mathematics, Poznan, August 1983.

[3] Baaz, M.: Infinite-valued Gödel Logics with 0-1 Projections and Relativizations, GÖDEL96Logical Foundations of Mathematics, Computer Science and Physics (P. Hájek, Ed.), 6, Springer-Verlag, Berlin, 1996.

[4] Banerjee, M., Chakraborty, K.: Algebras from rough sets, in: Rough-Neural Computing (S. Pal, A. Skowron, L. Polkowski, Eds.), Springer-Verlag, 2004, 157-188.

[5] Banerjee, M., Chakraborty, M.: Rough Sets Through Algebraic Logic, Fundamenta Informaticae, 28, 1996, 211-221.

[6] Bartušek, T., Navara, M.: Conjunctions of many-valued criteria, Proceedings of the International Conference Uncertainty Modelling'2001 (M. Komorníková, R. Mesiar, Eds.), Slovak Technical University, Bratislava, Slovakia, 2001.

[7] Belluce, L. P.: Generalized fuzzy connectives on MV-algebras, J. Math. Anal. Appl., 206, 1997, 485-499.

[8] Bonikowski, Z.: A Certain Conception of the Calculus of Rough Sets, Notre Dame journal of Formal Logic, 33, 1992, 412-421.

[9] Bonikowski, Z., Bryniarski, E., Wybraniec-Skardowska, U.: Extension and intentions in the rough set theory, Journal of Informations Sciences, 107, 1998, 149-167.

[10] Brown, L., Diker, M.: Ditopological texture spaces and intuitionistic sets, Fuzzy Sets and Systems, 98, 1998, 217-224.

[11] Cattaneo, G.: Generalized rough sets (preclusivity fuzzy-intuitionistic BZ lattices), Studia Logica, 58, 1997, 47-77.

[12] Cattaneo, G., Ciucci, D.: Theoretical Aspects of Shadowed Sets, in: Handbook of Granular Computing (W. Pedrycz, A. Skowron, V. Kreinovich, Eds.), John Wiley \& Sons, Chichester, England, 2008, 603-627.

[13] Cattaneo, G., Ciucci, D., Giuntini, R., Konig, M.: Algebraic Structures Related to Many Valued Logical Systems. Part I: Heyting Wajsberg Algebras, Fundamenta Informaticae, 63(4), 2004, 331-355.

[14] Cattaneo, G., Ciucci, D., Giuntini, R., Konig, M.: Algebraic Structures Related to Many Valued Logical Systems. Part II: Equivalence Among some Widespread Structures, Fundamenta Informaticae, 63(4), 2004, 357-373.

[15] Cattaneo, G., Giuntini, R., Pilla, R.: BZMV ${ }^{d M}$ and Stonian MV algebras (Applications to fuzzy sets and rough approximations), Fuzzy Sets and Systems, 108, 1999, 201-222.

[16] Cattaneo, G., Nisticò, G.: Brouwer-Zadeh Posets and Three valued Łukasiewicz posets, Fuzzy Sets and Systems, 33, 1989, 165-190. 
[17] Ciucci, D.: Approximation algebra and framework, Fundamenta Informaticae, 94(2), 2009, $147-161$.

[18] Ciucci, D.: Rough Approximations of Fuzzy Sets and related paradigms, in: Emerging Paradigms in Informatics, Systems and Communication (C. Batini, R. Schettini, Eds.), vol. 1 of Quaderni - Department of informatics, system and communication, Starrylink, 2009, 129-134.

[19] Ciucci, D., Dubois, D.: Implications and conjunctions in three valued structures. Application to rough sets, 2010, In preparation.

[20] Ciucci, D., Dubois, D.: Rough Sets and Three valued Connectives - Extended Abstract, Journal of Zhejiang Ocean University, 29, 2010, 411-415, Proceedings RST.

[21] Ciucci, D., Dubois, D.: Truth-Functionality, Rough Sets and Three-Valued Logics, Proceedings ISMVL, 2010.

[22] Coker, D.: A note on intuitionistic sets and intuitionistic points, Turkish Journal of Mathematics, 20, 1996, 343-351.

[23] Deschrijver, G., Kerre, E.: On the position of intuitionistic fuzzy set theory in the framework of theories modelling imprecision, Information Sciences, 177(8), 2007, 1860-1866.

[24] Dost, S., Brown, L.: Intuitionistic Textures Revisited, Hacettepe Journal of Mathematics and Statistics, 34S, 2005, 115-130, Special Issue Dedicated to the memory of D. Coker.

[25] Dubois, D.: On degrees of truth, partial ignorance and contradiction, Proceedings IPMU08 (L. Magdalena, M. Ojeda-Aciego, J. Verdegay, Eds.), 2008.

[26] Dubois, D., Prade, H.: Twofold fuzzy sets and rough sets - Some issues in knowledge representation, Fuzzy Sets and Systems, 23, 1987, 3-18.

[27] Dubois, D., Prade, H.: Conditional Objects as Nonmonotonic Consequence Relationships, IEEE Transaction of Sysyems, Man, and Cybernetics, 24(12), 1994, 1724-1740.

[28] Dubois, D., Prade, H.: An Introduction to Bipolar Representations of Information and Preference, International Journal of Intelligent Systems, 23, 2008, 866-877.

[29] Dubois, D., Prade, H.: Formal Representation of Uncertainty, in: Decision-making process (D. Bouyssou, D. g, M. Pirlot, H. Prade, Eds.), chapter 3, Wiley, 2009, 85-156.

[30] Dubois, D., Prade, H., Esteva, F., Godo, L.: An information-based discussion of vagueness:six scenarios leading to vagueness, in: Handbook of Categorization in Cognitive Science (H. Cohen, C. Lefebvre, Eds.), chapter 40, Elsevier Ltd., 2005, 891-909.

[31] Gehrke, M., Walker, E.: On the structure of Rough Sets, Bulletin Polish Academy of Science (Mathematics), 40, 1992, 235-245.

[32] Gentilhomme, M. Y.: Les ensembles flous en linguistique, Cahiers de linguistique theoretique et applique, Bucarest, 47, 1968, 47-65.

[33] Hájek, P.: Metamathematics of Fuzzy Logic, Kluwer, Dordrecht, 1998.

[34] Inuiguchi, M.: Generalizations of Rough Sets and Rule Extraction, Transaction on Rough Sets I, LNCS 3100, 2004, 96-119.

[35] Iwinski, T.: Algebras for rough sets, Bulletin of the Polish Academy of Sciences, series: Mathematics, 35, 1987, 673-683.

[36] Katzberg, J., Ziarko, W.: Variable Precision Extension of Rough Sets, Fundamenta Informaticae, 27(2,3), 1996, 155-168.

[37] Marek, V., Truszczynski, M.: Contributions to the Theory of Rough Sets, Fundamenta Informaticae, 34, 1999, 1-21.

[38] Monteiro, A.: Sur Les Algèbres de Heyting symétriques, Portugaliae Mathematica, 39, 1980, $1-237$.

[39] Narin'yani, A. S.: Sub-definite set - New data type for knowledge representation, Memo 4232, Computing Center, Novosibirsk, Russia, 1980, (in Russian, with an English summary).

[40] Pawlak, Z.: Information Systems - Theoretical Foundations, Information Systems, 6, 1981, 205-218.

[41] Pawlak, Z.: Rough sets and fuzzy sets, Fuzzy Sets and Systems, 17, 1985, 99-102.

[42] Pawlak, Z., Skowron, A.: Rough sets: Some extensions, Information Sciences, 177, 2007, 28-40.

[43] Pawlak, Z., Skowron, A.: Rudiments of rough sets, Information Sciences, 177, 2007, 3-27.

[44] Pedrycz, W.: Shadowed Sets: Representing and Processing Fuzzy Sets, IEEE Transaction on Systems, Man and Cybernetics - PART B: Cybernetics, 28(1), 1998, 103-109.

[45] Pedrycz, W.: Shadowed Sets: Bridging Fuzzy and Rough Sets, in: Rough Fuzzy Hybridization (S. Pal, A. Skowron, Eds.), Springer-Verlag, Singapore, 1999, 179-199. 
[46] Pedrycz, W.: Granular Computing with Shadowed Sets, Proceedings RSDGRC'05, 3641, Springer-Verlag, Berlin, 2005.

[47] Pedrycz, W., Vukovich, G.: Granular Computing with Shadowed Sets, International Journal of Intelligent Systems, 17, 2002, 173-197.

[48] Polkowski, L., Skowron, A., Eds.: Rough Sets in Knowledge Discovery 1, vol. 18 of Studies in Fuzziness and Soft Computing, Physica-Verlag, Heidelberg, New York, 1998.

[49] Pomykala, J., Pomykala, J.: The Stone Algebra of Rough Sets, Bulletin of the Polish Academy of Sciences - Mathematics, 36(7-8), 1988, 495-507.

[50] Vakarelov, D.: Notes on N-Lattices and Constructive Logic with Strong Negation, Studia Logica, 36, 1977, 109-125.

[51] Walker, E.: Stone Algebras, Conditional Events, and Three Valued Logic, IEEE Transaction of Sysyems, Man, and Cybernetics, 24(12), 1994, 1699-1707.

[52] Yao, Y.: Interval-set algebra for qualitative knowledge representation, Proceedings of the Fifth international Conference on Computing and Information, 1993.

[53] Yao, Y.: Generalized rough set models, chapter 16, Vol. 18 of Polkowski and Skowron [48], 1998, 286-318.

[54] Yao, Y.: Relational interpretations of neighborhood operators and rough set approximation operators, Information Sciences, 111, 1998, 239-259.

[55] Yao, Y.: Probabilistic Rough Set Approximations, International Journal of Approximate Reasoning, 49, 2008, 255-271.

[56] Yao, Y.: Interval Sets and Interval-Set Algebras, Proceedings of the 8th IEEE International Conference on Cognitive Informatics, 2009.

[57] Yao, Y., Li, X.: Comparison of rough-set and interval-set models for uncertain reasoning, Fundamenta Informaticae, 27, 1997, 289-298.

[58] Ziarko, W.: Variable Precision Rough Sets Model, Journal of Computer and Systems Sciences, 43(1), 1993, 39-59.

Dipartimento di Informatica, Sistemistica e Comunicazione, Università di MilanoBicocca, Via Bicocca degli Arcimboldi 8, I-20126 Milano (Italy)

E-mail address: ciucci@disco.unimib.it 